\title{
PYODERMA GANGRENOSUM AFTER LUMPECTOMY AND MAMMOPLASTY RESPONDS TO THE USE OF ANTI-TNF MONOCLONAL ANTIBODY
}

Rebeca Neves Heinzen', Liliane Raupp Gomes Pizzato², Evandro Luiz Mitri Parente³, Ivanio Alves Pereira, Adriana Magalhães De Oliveira Freitas ${ }^{5}$

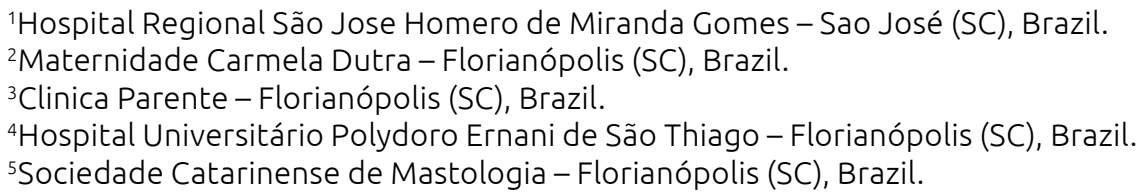

Introduction; Pyoderma gangrenosum $(\mathrm{PG})$ is a rare neutrophilic dermatosis of unknown etiology, which can be triggered after a surgical procedure. It can occur at any surgical site, but it is more common after breast surgeries. The trauma of the surgery can increase levels of inflammatory cytokines and result in a dysfunction of the innate immune system leading to a condition with cellulitis and dehiscence in the surgical wound. Despite PG being a rare condition, a differential diagnosis is important, because the debridement can exacerbate the condition and the antibiotics don't have clinical effects. We present a case report of PG after a breast surgery. Case report: A 37-year-old Caucasian female presented with a growing breast lump and the desire to reduce the size of her breasts. The woman had no comorbities, especially no history of autoimmune disorders. In 2009, she performed a primary mammoplasty in another service. At that time, she had a wound infection that resulted in an unfavorable aesthetic result. The patient underwent a lumpectomy it the right breast for a benign tumor (fibroadenoma) that was growing. It the same procedure, the plastic surgeon performed a second mammoplasty with a smooth prosthesis. Ten days later she presented with bilateral breast hyperemia, wound purulent discharge and dehiscence, without fever. As all breast lesions were sparing the nipple-areola complex, we had a strong suspicion of PG. She was treated with glucocorticoid prednisone 40mg once daily, colchicine $0.5 \mathrm{mg}$ twice daily and tumor necrosis factor (TNF) blocker 40mg subcutaneous every two weeks. After starting immunobiological therapy, she responded quickly with complete wound healing. Discussion:PG diagnosis was done based on clinical characteristics. It is important to be aware that this condition is frequently mistaken for a wound infection, but antibiotics do not alleviate the condition. Misdiagnosis could lead also to surgical management for wound debridement which would probably make lesions worse. Most PG starts after four days to six weeks post-operatively. In this case it started ten days after the surgery. The PG occurred after a benign breast surgery with mammoplasty, but it can also occur after breast cancer surgeries and reconstructions. Our patient did not have any disease associated with PG, such as inflammatory bowel disease, spondyloarthritis, rheumatoid arthritis, or hematologic malignancy. As reported in the literature, the PG was bilateral, but sometimes it can be unilateral. There have never been any reports describing involvement of the nipples and areolas. We presented a rare case of PG in a woman without risk factors who had a successful treatment with glucocorticoids. 